\section{THE PROBLEM OF GLIOMA OF THE BRAIN*}

BY

G. F. ROWBOTHAM, B.Sc., F.R.C.S.

Consultant in Neurological Surgery, No. 1 Regional Hospital Board

L. P. LASSMAN, M.B., B.S., F.R.C.S. Assistant Neurosurgeon, No. 1 Regional Hospital Board

A. C. JENKINS, M.D.

Demonstrator in Anatomy, University of Durham AND

MARJORIE E. BOUSFIELD

(From the Regional Centre of Neurological Surgery, General Hospital, Newcastle-upon-Tyne)

\section{[With Special Plate]}

There tends to be a periodic lack of interest in the study of cerebral gliomata, though these neoplasms continue to inflict their havoc on the health and lives of far too many young and middle-aged people. This paper is therefore aimed at recalling attention to this important subject and reviewing certain facts and conclusions gained from our experience with a consecutive series of 700 patients with neoplasms of the brain, of which 466 were gliomata.

\section{Surgical Pathology}

The gross post-mortem appearances of gliomata are usually so distinctive that these neoplasms can be differentiated almost as well by the naked eye as by histological examination. Of the 466 gliomata in our series, 294 were completely solid and 172 partly cystic. In only 20 of the cystic cases was the neoplasm composed of a large cyst and a relatively small amount of tumour; in the other 152 the cysts were small in relation to the solid mass of the tumour.

The gliomata were classified histologically according to cell type as originally suggested by Bailey and Cushing (1926), this work being carried out by the late A. F. Bernard Shaw, Professor of Pathology in the University of Durham, and also by Professor R. Paterson Smith. The results were as shown in Table I.

In the unclassified group were placed neoplasms the histological appearances of which did not strictly conform to the criteria of diagnosis laid down by Bailey and Cushing: most were highly malignant. Even the most typical of the astrocytomata and oligodendrogliomata were not composed of a uniform type of cell ; in the highly malignant types one zone differed considerably in cell content, cell size, mitotic activity, and vascularization. In no instance was it possible from the histological studies to reconstruct the general architecture of the growth and the way in which its mass was given cohesion. There were areas which seemed to be without any kind of histological pattern and yet did not appear to be zones of necrosis or of haemorrhage. A discrete edge to a glioma was never seen; brain tissue apparently reacts but little to the advance of a glioma. Cells presumably neoplastic in nature can nearly always be seen at considerable distances from, and entirely unconnected with, the main mass of the neoplasm.

*This paper was submitted for publication in December, 1952.
TABLE I

\begin{tabular}{|c|c|c|c|c|}
\hline \multicolumn{3}{|c|}{$\begin{array}{l}\text { Type of Glioma } \\
\text { (See Special Plate) }\end{array}$} & Adults & $\begin{array}{l}\text { Children } \\
\text { (Up to the Age } \\
\text { of 12) }\end{array}$ \\
\hline $\begin{array}{l}\text { Astrocytoma: } \\
\text { (a) Hemispheral } \\
\text { (b) Cerebellar } \\
\text { Astroblastoma } \\
\text { Spongioblastoma uni } \\
\text { Glioblastoma (spong } \\
\text { Oligodendroglioma } \\
\text { Ependymoma } \\
\text { Medulloblastoma } \\
\text { Haemangioblastoma } \\
\text { Pinealoma .. } \\
\text { Unclassified: } \\
\text { (a) Partly cystic } \\
\text { (b) Solid . } \\
\text { Papilloma } \quad .\end{array}$ & 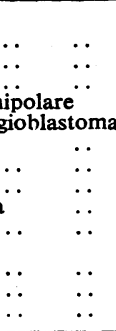 & 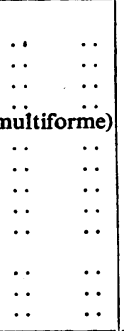 & $\begin{array}{r}54 \\
9 \\
10 \\
5 \\
76 \\
9 \\
7 \\
9 \\
5 \\
3 \\
66 \\
156 \\
2\end{array}$ & $\begin{array}{r}2 \\
6 \\
2 \\
2 \\
1 \\
\frac{1}{6} \\
21 \\
\frac{2}{2} \\
2 \\
11 \\
-\end{array}$ \\
\hline Total & $\ldots . \quad \ldots$ & .. & 411 & 55 \\
\hline
\end{tabular}

As the appearance of a cell after it has been prepared for histological examination cannot possibly resemble that of its natural state, an effort was made to grow specimens in culture. This work was first carried out at the Christie Hospital and Holt Radium Institute, Manchester, with the help of Dr. Edith Ralston Patterson. The accompanying drawing, made by Miss D. Davison, depicts what was seen through a microscope at the edge of a culture of a glioblastoma.

There is now a tendency to classify gliomata according to their degree and type of malignancy. Theoretically this method may be an advance on the classification by cell type ; in practice, however, it is doubtful whether it has any advantages, because, although precise criteria for classification can be laid down, tumour variations are so great that strict grouping according to the accepted rulings is difficult.

In the 231 cases in which the glioma was given a formal name the sites of occurrence were: frontal lobe, 49; temporal lobe, 66 ; parietal lobe, 44 ; occipital lobe, 12 ; third ventricle, 8 ; fourth ventricle, 6 ; brain-stem, 5 ; cerebellum, 41 . In some instances the neoplasm was too large to be certain of the structure from which it originated, although it is obvious that most of the malignant types arose in the basal ganglia, vermis of the cerebellum, or corpus callosum. Also, although the bulk of many neoplasms occupied one lobe of the brain, extension had taken place into neighbouring zones. In no instance was there a metastasis outside the central nervous system.

The age incidence was as follows: 1 to 12 years, 42 ; 13 to 19,$18 ; 20$ to 29,$31 ; 30$ to 39,$43 ; 40$ to 49,44 ; 50 to 59,$44 ; 60$ to 69,9 .

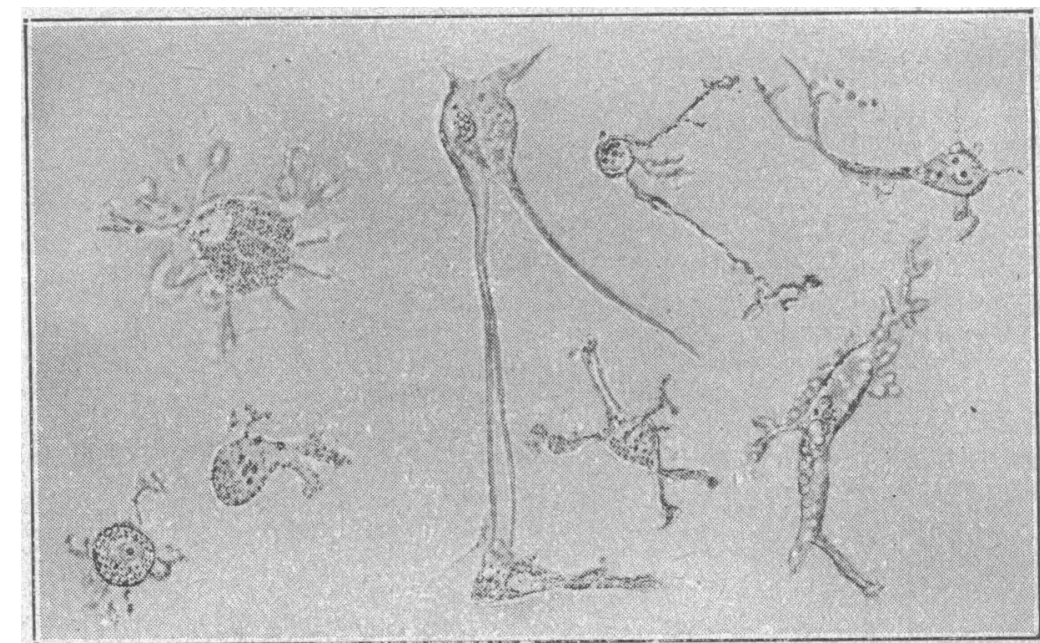

The microscopical aspect of cells growing from the edge of a culture of a glioblastoma. These appearances no doubt conform to the normal characteristics of cells before subjection to the processes of histological preparation. 


\section{The Problem of Early Diagnosis}

In a case of cerebral glioma the clinical picture of course depends substantially on whether the neoplasm is in the early or the late stages of development. Moreover, in view of the many and widespread structures from which a glioma may arise, it will be obvious how difficult it is to consider a diagnosis without first grouping these neoplasms according, to type and then subgrouping the types according to site of origin. For example, the clinical picture of cystic astrocytoma of the cerebellum is very different from that of astrocytomatous cyst of a hemisphere. Nevertheless this apparent need for subgrouping leads to a tendency to await a fully developed clinical syndrome before a firm diagnosis of cerebral neoplasm is risked. Late diagnosis may not be greatly detrimental therapeutically where a glioma is concerned, but has serious consequences in the case of meningioma or acoustic neuroma.

Here, in the interests of earlier recognition of a neoplasm, we will attempt to discuss diagnosis from the first symptom or sign in the group as a whole, without segregating the tumours into subgroups-for example, cystic astrocytoma of the cerebellum and the glioblastoma of the hemispheres. In the 231 cases in which the neoplasm was given a formal name we were able to classify the initial symptoms or signs as follows:

1. Rise in Intracranial Pressure.-In 142 instances headache, vomiting, dizziness, and papilloedema, singly or in combination, were the first signs or symptoms. Papilloedema was an early sign in 10 cases only, and, of course, was pathognomonic of a space-occupying lesion. In the remaining 89 cases a diagnosis was impossible on the characteristics of the headache, dizziness, or vomiting.

2. Epilepsy.-A sudden attack of epilepsy was the first sign in 40 cases; in 38 of these the seizures were major, and in the remaining 2 focal. All the attacks occurred in patients between the ages of 20 and 60 years. In 6 cases the seizures were followed by neurological signs from which the patient never fully recovered; in 4 cases there was hemiparesis, and in 2 a mild dysphasia. In every instance a lesion other than a glioma, or even a neoplasm, could have been the cause.

3. Mental Change.-Although in only 15 out of the 231 cases did mental change herald the presence of a cerebral glioma, it is probable that in many cases this symptom was overlooked or its significance not appreciated. Initially, mental change leads to minor and very subtle changes in behaviour that can be elicited only by direct questioning of an intimate relative or friend. A common first sign is a carelessness about personal appearance-for example, a man hitherto fastidious about his appearance may fail to shave cleanly, or a woman usually scrupulous about her hair may leave it unbrushed and untidy; again, a normally punctual person may start to get up late and show no concern about prompt arrival at work. Every change in behaviour cannot, of course, be regarded as having a sinister meaning. An intimate relative is quick to detect deterioration, but often withholds the information if not directly cross-questioned. The assessment of a minor change in behaviour makes heavy calls on the good judgment and experience of a general practitioner. Frank mental confusion and gross faults in behaviour, such as incontinence, are later signs of mental deterioration. Mental signs may, of course, be due to many kinds of pathological change, and when present can merely call attention to the possibility of a cerebral tumour and no more.

4. Abnormal Neurological Signs.-The appearance and persistence of an abnormal neurological sign was the first evidence of neoplasm in 34 of the 231 cases. All these signs, some of which developed rapidly and some slowly, could obviously have been caused by a lesion other than a tumour. They are as follows: hemiparesis, in 16 cases ; dysphasia, 9 ; squint, 4 ; sensory loss, 2 ; ataxia, 3 .

\section{Differential Diagnosis}

It will be apparent, therefore, that in most cases of glioma it is impossible to make a diagnosis on the first symptom or sign. Often it is difficult even to decide whether or not the brain itself is primarily affected. Also, to make diagnosis more difficult, other space-occupying lesions present more or less in the same way. In our series, for example, the gliomata had to be differentiated not only from vascular and degenerative lesions of the central nervous system, but from other types of space-occupying lesion, as shown in Table II.

\section{TABLE II}

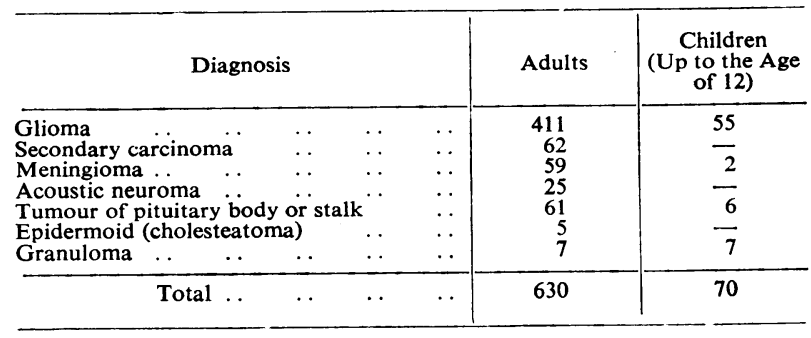

In the few instances when the first symptom or sign throws strong suspicion on the possibility of a cerebral neoplasm it is probably wiser to proceed with special neurological investigations to establish diagnosis than to await the development of further confirmatory symptoms or signs. In the majority of cases, however, it is necessary to await possible developments before it can be decided that one is dealing with a progressive lesion of the central nervous system. But it is absolutely wrong to await the development of a fully established clinical syndrome before making a diagnosis or applying special diagnostic tests. For example, it is not necessary, in the case of a man who is forgetting an occasional word, to defer a diagnosis of glioblastoma until he is completely aphasic, disorientated, incontinent, and going blind with papilloedema. At the same time to swing violently from the policy of overwaiting to mere mechanistic methods of diagnosis is equally unsatisfactory. Obviously a multitude of patients with minor and purely transient disorders should not be subjected to the discomforts and dangers of special neurological investigations, nor should the usual simple clinical tests to eliminate conditions such as nephritis and hypertension be omitted.

\section{Special Diagnostic Methods}

Although straight radiographs of the head rarely reveal anything abnormal in the early stages of a space-occupying mass in the brain, they do occasionally show signs of pressure, hyperostosis, erosion, calcification, or dislocation of the pineal gland, and therefore should be made use of, particularly as this form of radiography is painless and without danger. Electroencephalographic investigation is also painless and without risk, and should be used routinely in the detection of neoplasms in their early stages. We have found electroencephalography to have limited value, however, as it tends to establish that a physical lesion is present without giving precise information concerning its pathology ; but this should not preclude its free use.

Lumbar puncture may show that intracranial pressure is raised before there is clinical evidence of it. Occasionally a rise in the protein content of the cerebrospinal fluid is the first positive evidence of a cerebral tumour. In the early stages of a space-occupying lesion the value of lumbar puncture far outweighs its possible dangers.

When, in spite of the procedures referred to above, diagnosis is still in doubt, air encephalography, ventriculography, or cerebral angiography will be necessary to decide whether a neoplasm is present, and, if so, its nature. 
In our experience, where there is little evidence of a rise in intracranial pressure, air encephalography is perfectly safe and is one of the most reliable methods for detecting a space-occupying lesion of the brain. Although we have performed air encephalography many hundreds of times during the last ten years we have not had one fatality or seen a single complication as a result.

Ventriculography is the safer method of air or oxygen replacement when there is obvious evidence that the intracranial pressure is raised. This procedure involves making two holes in the skull through which cannulae can be passed into the lateral ventricle, whereas with air encephalography it is necessary only to tap the spinal theca with a hollow needle at the lumbar or cisterna magna level.

Cerebral angiography is a most important procedure, by the aid of which it is possible not only to determine with great precision whether a space-occupying lesion is present and, if so, its location, but also to obtain valuable information concerning its pathology.

Neither cerebral angiography nor ventriculography is to be carried out lightly, as considerable danger is involved.

\section{Operative Treatment}

It is our purpose to summarize briefly the surgical methods of treatment we have employed in this series of 466 cases of glioma, of which 405 were operated upon, and to review our main surgical conclusions.

1. When once a cerebral tumour has been firmly diagnosed in its early stages, and provided the intracranial pressure is not high, no effort should be spared to establish its precise location and pathological nature, if necessary by air studies and angiography.

2. Burr-hole inspection and probing we have found to be useful in diagnosis for excluding chronic subdural haematoma or intracranial abscess, but taking a biopsy specimen through a burr-hole we have found to be of little value and often dangerous.

3. In the later stages of a cerebral neoplasm, when the intracranial pressure has been obviously high, we have often dispensed with air studies or angiography and chosen the site for exploration on the evidence of clinical symptoms and signs. Nowadays the removal of an osteoplastic flap for exploration of a tumour through the dura mater is a relatively benign procedure; it is often safer, quicker, and less dangerous to the patient than ventriculography or angiography.

4. To plan an exploration over the vault of the skull or in the posterior fossa merely to uncover a tumour without also taking into account the anatomical peculiarities of the area concerned will usually lead to unnecessary technical complications ; for example, radical operations in the area of the frontal fossa are difficult unless the osteoplastic flap has been cut close to the edge of the superior longitudinal sinus and down to the floor of the frontal fossa. In operations in the area of the posterior fossa an angle tumour cannot be approached adequately through a midline exposure.

5. When operating to remove one of the more benign types of glioma the excision should be as radical as possible but without inflicting damage on those areas of the brain that must inevitably lead to severe incapacity. For example, we regard the surgical imposition of a hemiplegia as worse than failure.

6. Experience has taught us that piecemeal removal of a glioma is a waste of time. The only worthwhile type of internal decompression is the formal resection of a block of cerebral tissue-for example, excision of that part of the frontal lobe of the brain that lies within the anterior fossa.

7. Subtemporal decompression is to be avoided at all costs. For the last ten years it has been our practice, even if an osteoplastic flap is raised, never to open the dura mater unless there is reasonable certainty that it can be closed again; in other words, operability must be deter- mined by the appearance of the brain through the dura mater or by probing. First to open the dura in the hope of being able to do something useful operatively will in only too many cases lead to the development of unsightly bulges on the head due to cerebral herniations.

8. When a diagnosis of malignant glioma can be established beyond reasonable doubt, a surgeon is justified in refraining from operating. With the help of special investigations, particularly cerebral angiography, a reasonably certain diagnosis can often be arrived at.

\section{Prognosis}

It will have been seen from the section on surgical pathology that every glioma is malignant. Indeed, the majority of gliomata are highly malignant and grow so quickly that they cause serious incapacity within a few months, and death within two years. A small percentage of neoplasms at the astrocytomatous end of the scale are relatively benign and may continue to grow for 10 to 20 years before fatally compressing the brain-stem. Some tumours, such as the cystic gliomata and haemangioblastomata of the cerebellum, although not naturally self-limiting, are so circumscribed that they can be totally excised and the patient cured. However, in the large majority of cases surgery cannot offer cures, and, this being so, it might with reason be asked why a neurological surgeon so often operates on the gliomata. Indeed, the taunt that his persistent efforts at surgical cure in these cases reveal "the triumph of optimism over common sense" has often been levelled. There is no doubt to prolong a patient's life in misery is poor surgical judgment. None the less, despite his knowledge and experience, a surgeon is often compelled to operate against his better judgment, by pressure of relatives or colleagues or by the uncertainty of the pathological diagnosis.

In reviewing the results of the operative treatment of gliomata a broad perspective must be maintained. For example, a patient's life can easily be prolonged by a wide but unsightly subtemporal decompression. In this connexion it might be asked what are the ethical responsibilities of a surgeon. His main object is, of course, to cure the patient or to relieve severe symptoms which are unaffected by simpler methods. However, a surgeon is quite entitled not to interfere with natural processes where he is confident he cannot favourably alter their course by his intervention. The more experienced a surgeon becomes the less frequently is he inclined to operate on gliomata.

\section{Results}

The results in our series have been as follows :

Cures.-Seven patients have been completely cured; in this group were 2 cases of haemangioblastoma and 3 of cystic astrocytoma of the cerebellum, and 2 of mucous cyst of the third ventricle.

Substantial Prolongation of Useful Life.-Twenty-three patients who were already completely incapacitated by the effects of cerebral compression were returned to useful life for the following periods : 4 with cystic glioma of the cerebellum, 5 years; 2 with oligodendroglioma of the frontal lobe, 5 years ; 10 with cystic astrocytoma of a hemisphere, 2 years; and 7 with oligodendroglioma of a hemisphere, 2 years.

Substantial Relief of Symptoms.-In 52 cases distressing symptoms, such as severe pain and intractable vomiting, were relieved by operation when drugs, other than those interfering with consciousness, were of no avail.

Deep $x$-ray therapy, although found to retard the rate of growth of some of the more highly malignant of the gliomata, was never curative.

\section{Conclusions}

Though surgery has little to offer in the cure of cerebral gliomata, the diagnosis of these neoplasms, the care of patients suffering from them, and the management of near 
relatives still remain the onerous responsibility, and the duty, of those doctors who choose to make the problem of cerebral neoplasia one of their primary interests. Moreover-and this is even more important-in our series of 700 unselected cases of cerebral neoplasm, although 466 were gliomata, there were 147 cases that were potentially curable by surgery; in other words, $21 \%$ of favourable cases. In cases of neoplasm of the brain the segregation of the favourable cases from those of glioma in the early stages is one of the main problems of differential diagnosis, and carelessness in the diagnosis and handling of the glioma cases will reflect unfavourably on the potentially curable group.

In a further group of cases we are already using the newer methods of diagnosis, such as with radioactive isotopes, and are experimenting with biochemical forms of treatment and deep $x$-ray therapy.

\section{Summary}

Experience with 700 cerebral neoplasms, of which 466 were gliomata, is reviewed.

The majority of these new growths are highly malignant and lead to death within two years. Early diagnosis is important. The initial symptoms and signs are raised intracranial pressure, epilepsy, mental change, and neurological signs, though it is generally. impossible to base a diagnosis solely on the clinical evidence. $X$ rays, electroencephalography, and lumbar puncture are useful diagnostic aids, but when these fail air encephalography, ventriculography, or angiography will be necessary to decide if a space-occupying lesion is present.

Surgical methods of treatment are summarized and discussed. It is emphasized that subtemporal decompression should be avoided.

Of the 405 patients operated upon, 7 were cured, 23 were returned to a useful life for periods of two to five years, and 52 relieved of distressing symptoms such as severe pain and vomiting.

The preparation of this work was assisted by a Research Grant from No. 1 Regional Hospital Board.

\section{REFERENCE}

Bailey, P., and Cushing, H. W. (1926). A Classification of the Tumours of the Glioma Group on a Histogenic Basis with a Correlated Study of Prognosis. Lippincott, Philadelphia.

The "Mental Health Facilities and Needs of Australia" were the subject of a report drawn up by Dr. Alan Stoller, Melbourne psychiatrist, with the assistance of Mr. K. W. ARSCOTT. The report was undertaken at the instigation of the Commonwealth Government and published by it. Its scope included examination of mental hospitals and auxiliary services, mental deficiency programmes, psychiatric services, and State legislation. Dr. Stoller sums up his findings on the hospitals: "Mental hospitals are hopelessly overcrowded, poorly maintained, and short-staffed. ... Diagnostic and treatment equipment and facilities are sorely lacking. The hospitals are in no position to develop out-patient, training, and educational programmes, and research is virtually unthinkable." The average overcrowding percentage was $20 \%$-at the main mental hospital for Queensland the figure was $95 \%$. Victoria had the best mental health facilities, with active voluntary bodies, and residential centres for defective children. New South Wales provided four psychiatric clinics through the school medical service; elsewhere help for retarded children was extremely limited and legislation for them incomplete, so that often the number of such children was unknown. Early in May the Commonwealth Government announced a fA.30m. capital works programme to improve mental health facilities throughout the country.

\section{BERYLLIOSIS : A CASE REPORT}

BY

I. B. SNEDdon, M.B., Ch.B., M.R.C.P. Consultant Dermatologist, Rupert Hallam Department of Dermatology, Sheffield

The hazards of dermatitis and respiratory disease from handling beryllium compounds were known in Europe before the last war, and Marradi Fabroni (1935) suggested calling the lung disease berylliosis. Various syndromes caused by beryllium were seen in considerable numbers in the United States of America during the last war, and these have been reviewed by DeNardi et al. (1953). In Great Britain, beryllium has been used, though not in the same quantities as in America, in the fluorescent lamp industry and in the form of berylliumcopper alloys in the manufacture of electrical equipment and special springs. It is remarkable, therefore, that there has been only one published case of delayed pneumonitis in a beryllium worker in this country (Agate, 1948) and one case of beryllium granuloma of the skin in a fluorescent-light-tube worker (Lederer and Savage, 1954).

The delayed chemical pneumonitis first described by Hardy and Tabershaw (1946) is perhaps the most interesting and elusive manifestation of beryllium toxicity. It is a slowly progressive sarcoid-like change in the lungs and liver which may come on after a very short exposure to beryllium compounds and after a latent period which may vary from a few months to eleven years. The clinical features are the insidious onset of weight loss, weakness, dyspnoea, and cough, with occasional febrile periods. The prognosis is grave, as the mortality in 35 cases reported by DeNardi et al. (1953) was 35\%, and many of the remainder were severely disabled by pulmonary fibrosis.

The only British case (Agate, 1948) occurred in a physicist working on the development of tubular fluorescent lamps who was exposed to small quantities of zinc beryllium manganese. His symptoms did not develop until three years after his last contact with beryllium.

Although the first cases were described in a fluorescent lamp factory, it has also been reported in a brass foundry making and finishing beryllium-copper alloys (Jackson, 1950), and even in people who lived near beryllium extraction plants. Hardy (1951) lists 11 different industries in which chronic beryllium poisoning has been found. The incidence of chronic berylliosis is fortunately low. DeNardi et al. (1953) report 8 cases among 3,027 workers in three beryllium extraction plants, though 6 cases were found in 310 workers in the brass foundry (Jackson, 1950).

The following case is reported to draw attention to the possibility of delayed chemical pneumonitis due to beryllium in an industry hitherto regarded as safe. The case also shows the combination of unusual skin granulomata with lung lesions and would appear to be due to beryllium-copper alloy.

\section{Case Record}

A 25-year-old woman was referred to the skin department in August, 1954, for treatment of Raynaud's phenomenon of the fingers, from which she had suffered since the age of 10 . It was noted that, as well as having cold blue fingers, she had numerous linear granulomatous papules on the fingers, palms, forearms, and legs (Fig. 1), and that she had a granulating wound on the dorsum of the left foot, which she 


\section{G. F. ROWBOTHAM ET $A L$. : CEREBRAL GLIOMA}

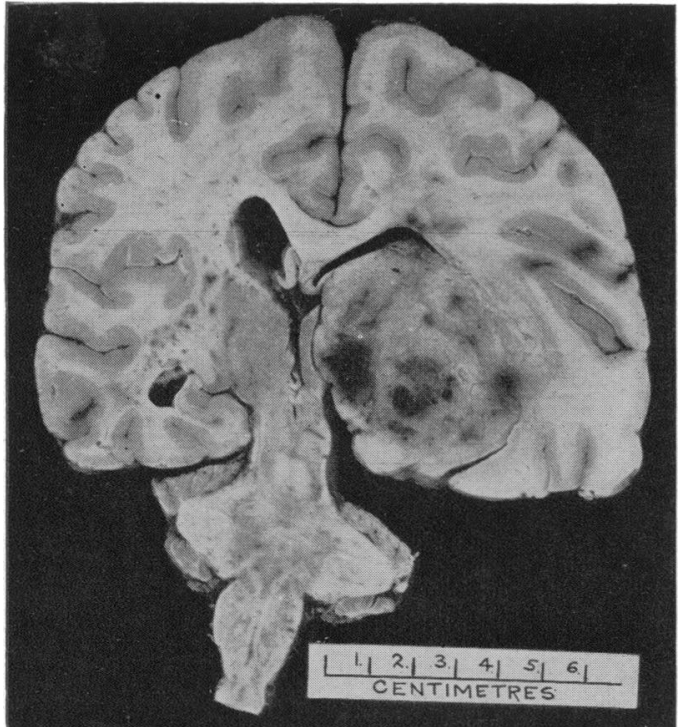

Glioblastoma of basal ganglia.

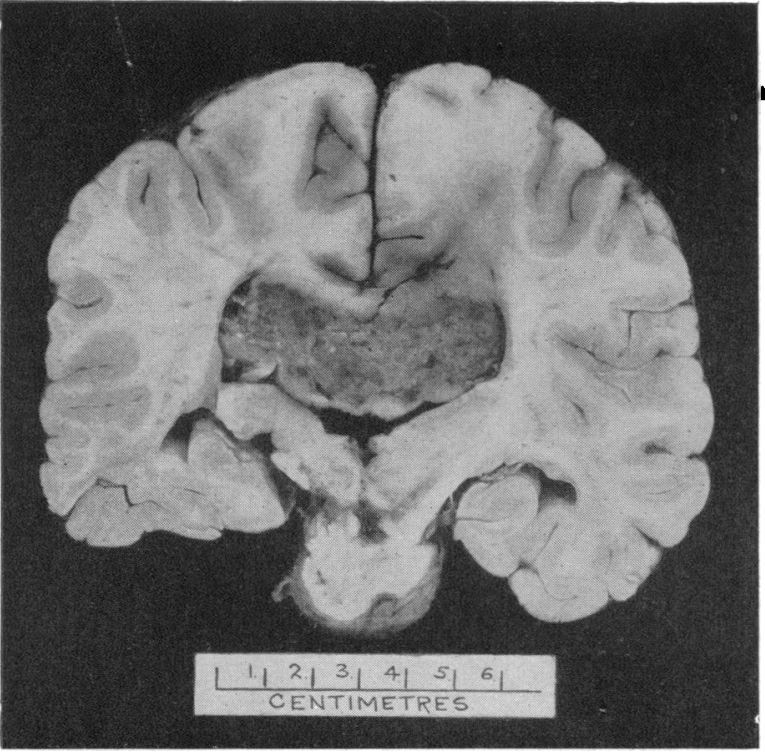

Glioma of corpus callosum.

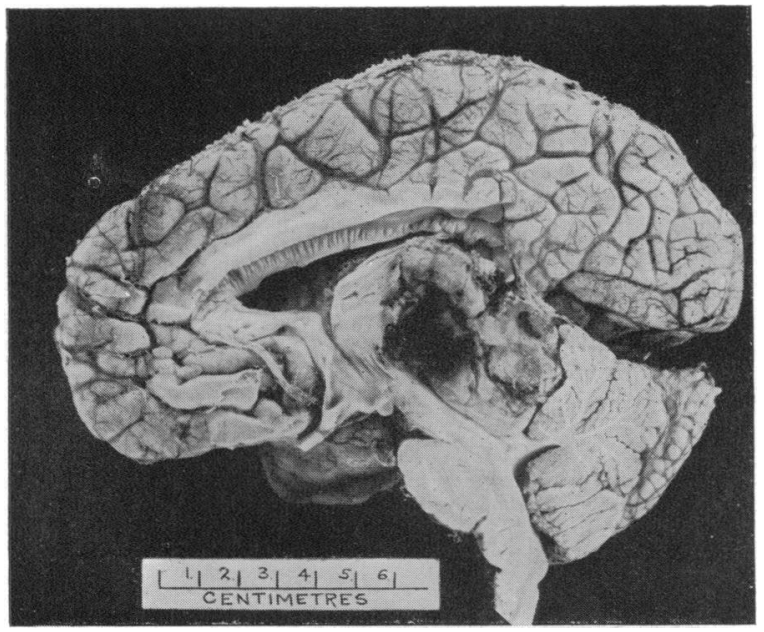

Pinealoma.

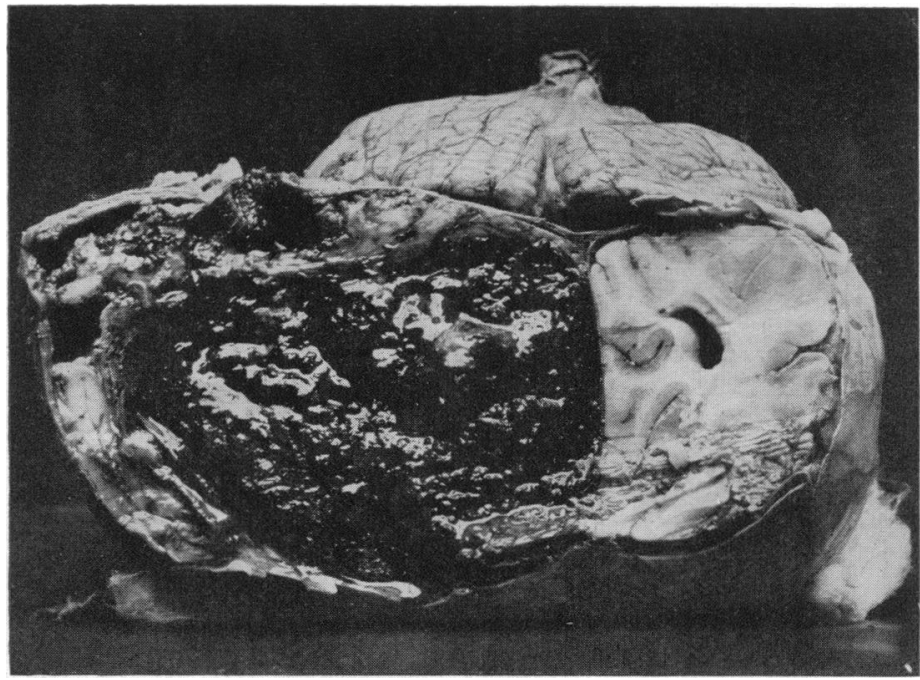

Rapidly growing malignant glioma.

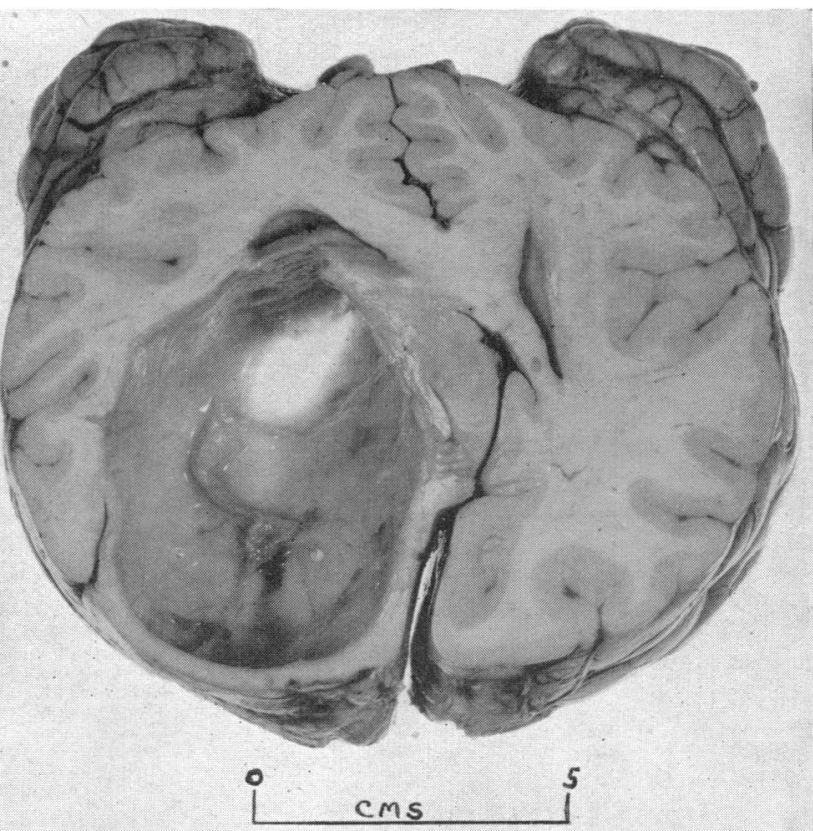

Astrocytomatous cyst of a hemisphere.

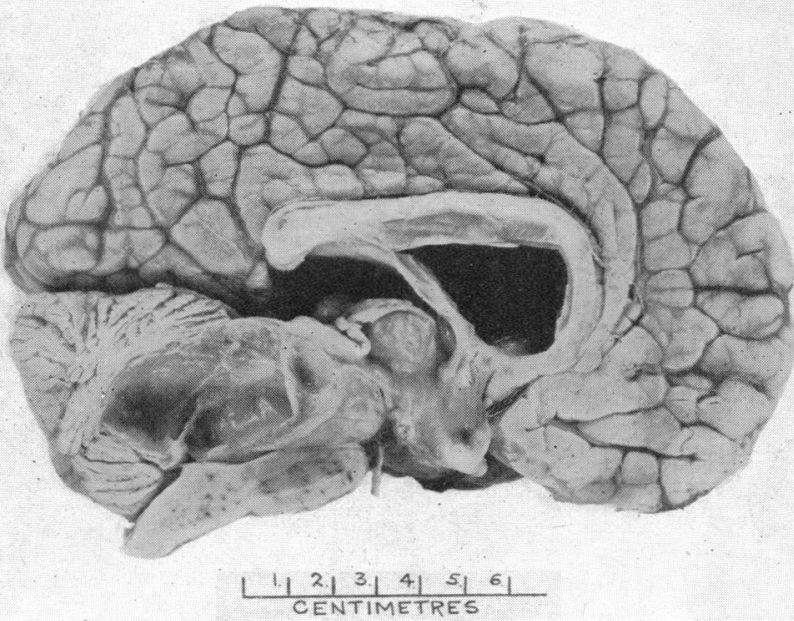

Cystic astrocytoma of cerebellum. 